Article

\title{
Bioinformatics Analysis of Phylogeny and Transcription of TAA/YUC Auxin Biosynthetic Genes
}

\author{
Axel Poulet 1,2 (D) and Verena Kriechbaumer ${ }^{1, *}$ \\ 1 Department of Biological and Medical Sciences, Oxford Brookes University, Oxford OX3 0BP, UK; \\ axel.poulet@emory.edu \\ 2 Department of Biostatistics and Bioinformatics, Rollins School of Public Health, Emory University, Atlanta, \\ GA 30322, USA \\ * Correspondence: vkriechbaumer@brookes.ac.uk; Tel.: +44(0)1865-48-8403
}

Received: 20 June 2017; Accepted: 13 August 2017; Published: 18 August 2017

\begin{abstract}
Auxin is a main plant growth hormone crucial in a multitude of developmental processes in plants. Auxin biosynthesis via the tryptophan aminotransferase of arabidopsis (TAA)/YUCCA (YUC) route involving tryptophan aminotransferases and YUC flavin-dependent monooxygenases that produce the auxin indole-3-acetic acid (IAA) from tryptophan is currently the most researched auxin biosynthetic pathway. Previous data showed that, in maize and arabidopsis, TAA/YUC-dependent auxin biosynthesis can be detected in endoplasmic reticulum (ER) microsomal fractions, and a subset of auxin biosynthetic proteins are localized to the ER, mainly due to transmembrane domains (TMD). The phylogeny presented here for TAA/TAR (tryptophan aminotransferase related) and YUC proteins analyses phylogenetic groups as well as transmembrane domains for ER-membrane localisation. In addition, RNAseq datasets are analysed for transcript abundance of YUC and TAA/TAR proteins in Arabidopsis thaliana. We show that ER membrane localisation for TAA/YUC proteins involved in auxin biosynthesis is already present early on in the evolution of mosses and club mosses. ER membrane anchored YUC proteins can mainly be found in roots, while cytosolic proteins are more abundant in the shoot. The distribution between the different phylogenetic classes in root and shoot may well originate from gene duplications, and the phylogenetic groups detected also overlap with the biological function.
\end{abstract}

Keywords: auxin; indole-3-acetic acid (IAA); endoplasmic reticulum (ER); transmembrane domain (TMD); phylogeny; RNAseq; YUCCA (YUC); tryptophan aminotransferase of arabidopsis (TAA); tryptophan aminotransferase related (TAR); gene duplication

\section{Introduction}

Auxin is the major plant growth hormone and is involved in most developmental processes in plants. The multiplicity of auxin biosynthetic pathways that can be independently and differentially regulated result in a metabolic network which is capable of rapid end efficient dynamic changes. To understand the mechanism of local auxin action, it is crucial to determine the regulation of auxin biosynthesis. The currently most-researched pathway of auxin biosynthesis is the tryptophan aminotransferase of arabidopsis (TAA)/YUCCA (YUC) route involving tryptophan aminotransferases and YUC flavin-dependent mono-oxygenases to produce the auxin indole-3-acetic acid (IAA) from tryptophan.

The two-step pathway from tryptophan (Trp) to IAA, catalysed by the TAA proteins and the YUC flavin-containing mono-oxygenases, is the first identified complete auxin biosynthetic pathway, which is essential for almost all of the major developmental events in plants [1]. In the TAA/YUC-route, TAA/TAR (tryptophan aminotransferase related) enzymes convert Trp to indole-3-pyruvic acid (IPyA) 
which is then converted by YUC proteins to IAA [2]. YUC enzymes were first identified as key auxin biosynthesis enzymes as the overexpression of YUC in arabidopsis leads to auxin overproduction [3]. Importantly YUC enzymes were also identified via the first ever auxin biosynthetic mutants [4]. Inactivation of the first step in TAA/YUC-dependent auxin biosynthesis (TAA1 and its close homologues TAR1 and TAR2) leads to partial auxin deficiency and defects in several developmental processes [5]. With 11 YUC genes and three TAA/TAR genes in arabidopsis, this important pathway shows a high redundancy. Nonetheless, the disruption of multiple YUC genes causes dramatic developmental defects mainly in roots; these root phenotypes can be rescued by adding auxin to growth media [6].

\subsection{Subcellular Location of Auxin Biosynthetic Genes}

We have previously showed that, in maize [7] as well as in arabidopsis [8], auxin biosynthetic activity can be found in microsomal fractions, and a subset of auxin biosynthetic proteins show endoplasmic reticulum (ER)-localisation, mainly due to transmembrane domains (TMD). This could indicate a model of auxin function using ER membrane localisation and subcellular compartmentation for additional layers of regulation, which raises a question about the localisation of all the components of the arabidopsis TAA/YUC route.

Arabidopsis appears to use two separate sets of $Y U C$ genes for auxin biosynthesis in roots and shoots. YUC1, 2, 4 and 6 are the main YUC proteins in shoots, and YUC3, 5, 7, 8 and 9 are responsible for producing auxin in roots [5]. This is underlined by mutant studies: yuc1/2/4/6 quadruple mutants have severe defects in vascular patterning and flower development but have no root defects, which is consistent with the expression patterns [6]. YUC3, 5, 7, 8 and 9 are expressed during root development and quintuple mutants of these five $Y U C$ genes (yucQ mutant) develop short and agravitropic root patterns [6]. The $y u c Q$ phenotypes are rescued by IAA addition or by expressing a $Y U C$ gene in yucQ roots but not by overexpression of a YUC gene in shoots [6]. This indicates that local auxin biosynthesis in roots is necessary for root development and that auxin transported from shoots is not sufficient. Interestingly, the shoot YUCs 1, 2, 4 and 6 are cytosolic, whereas YUCs located in roots (YUC5, 7, 8 and 9) are ER membrane-bound [8]. YUC3 is the only cytosolic YUC protein in the root. In contrast, arabidopsis seedlings appear to use the same set of TAA/TAR genes in both roots and shoots [5,9].

The ER is also interesting in terms of auxin transport, as recent data shows ER-specific PIN-FORMED (PIN) and PIN-LIKES (PILS) protein families of auxin transporters. Localization studies revealed that PIN5, PIN6, and PIN8 mainly localize to the ER [10-14].

As there is this apparent division between membrane and cytosolic proteins in roots and shoots, the evolution and the protein abundance of the different proteins is of interest.

We present here a phylogenetic analysis for TAA/TAR and YUC proteins that phylogenetic groups takes into account as well as transmembrane domains for ER-membrane localisation. Along with this, RNAseq datasets are mined to analyse the transcript abundance of YUC, TAA, and TAR proteins in Arabidopsis thaliana.

\section{Results}

\subsection{Phylogenetic Analysis of YUC Proteins}

The eleven YUC proteins in A. thaliana can be grouped in four different groups (Figure 1). Five proteins comprise the first group: YUC3, YUC5, YUC7, YUC8, and YUC9. Here, two smaller subgroups can be defined, with YUC3 and YUC7 grouping together and YUC5, YUC8 and YUC9 also grouping together. The three other groups are YUC1-YUC4, YUC2-YUC6 and YUC10-YUC11.

During the homologous protein detection, 187 homologous YUC proteins were identified within all the species analysed (Table 1 and Table S1). The phylogenetic tree of these proteins (Figure 1A) shows the same topology to the tree for A. thaliana YUCs (AtYUCs) in Figure 1B. A comparison of these two trees in Figure 1 displays the same protein clusters: YUC10-YUC11, YUC1-YUC4, YUC2-YUC6 and 
YUC3-YUC7-YUC8-YUC5-YUC9. As in arabidopsis, ER-localised and cytosolic auxin biosynthetic proteins mainly group together. In this analysis, the prediction for TMD and protein domains was included in the analysis (InterProScan version 64.0, EMBL-EB, Hinxton, UK) [15]. This could give an indication of whether membrane localisation is a more ancient form —in evolutionary terms—or was added later on.

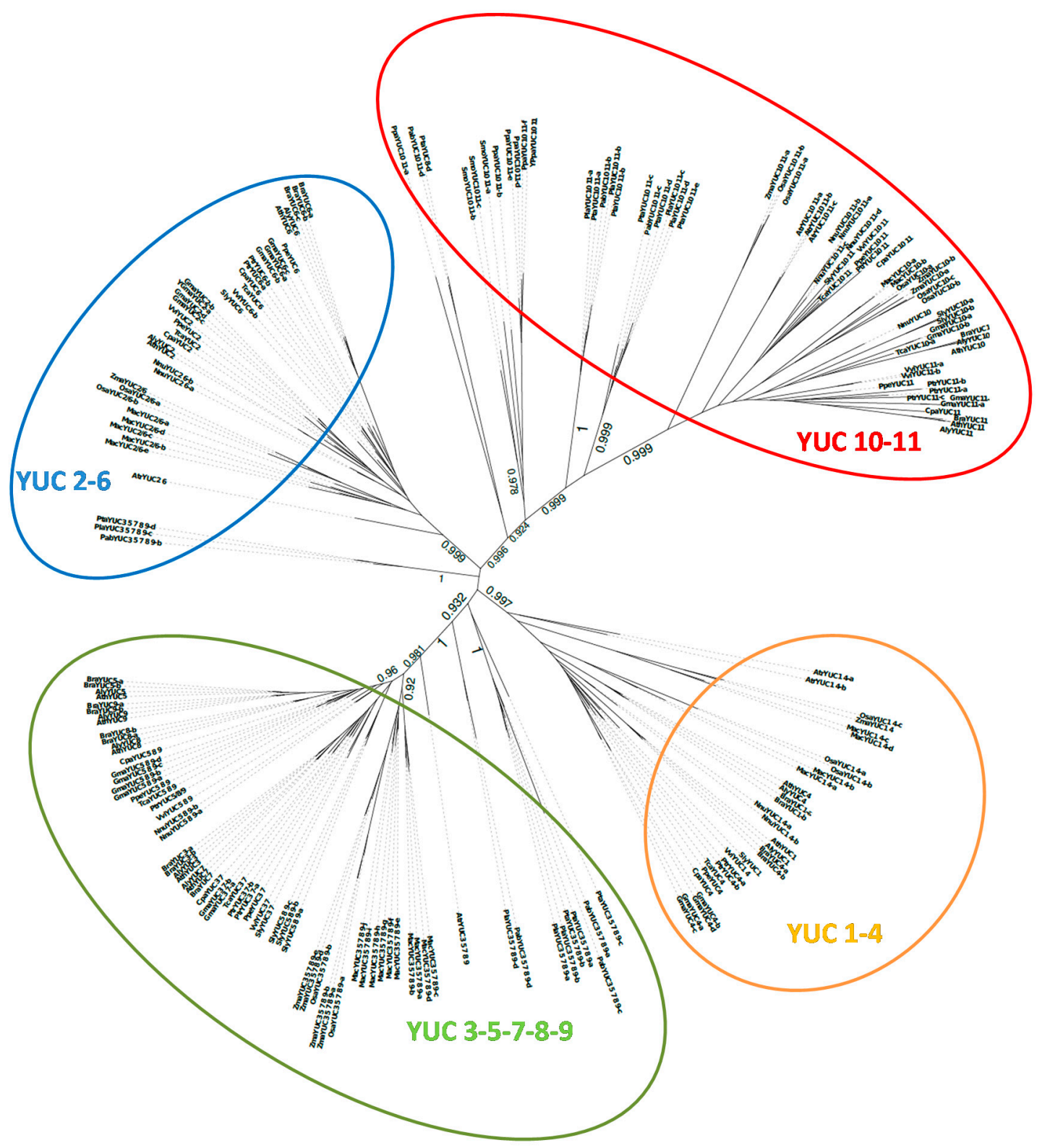

(A)

Figure 1. Cont. 


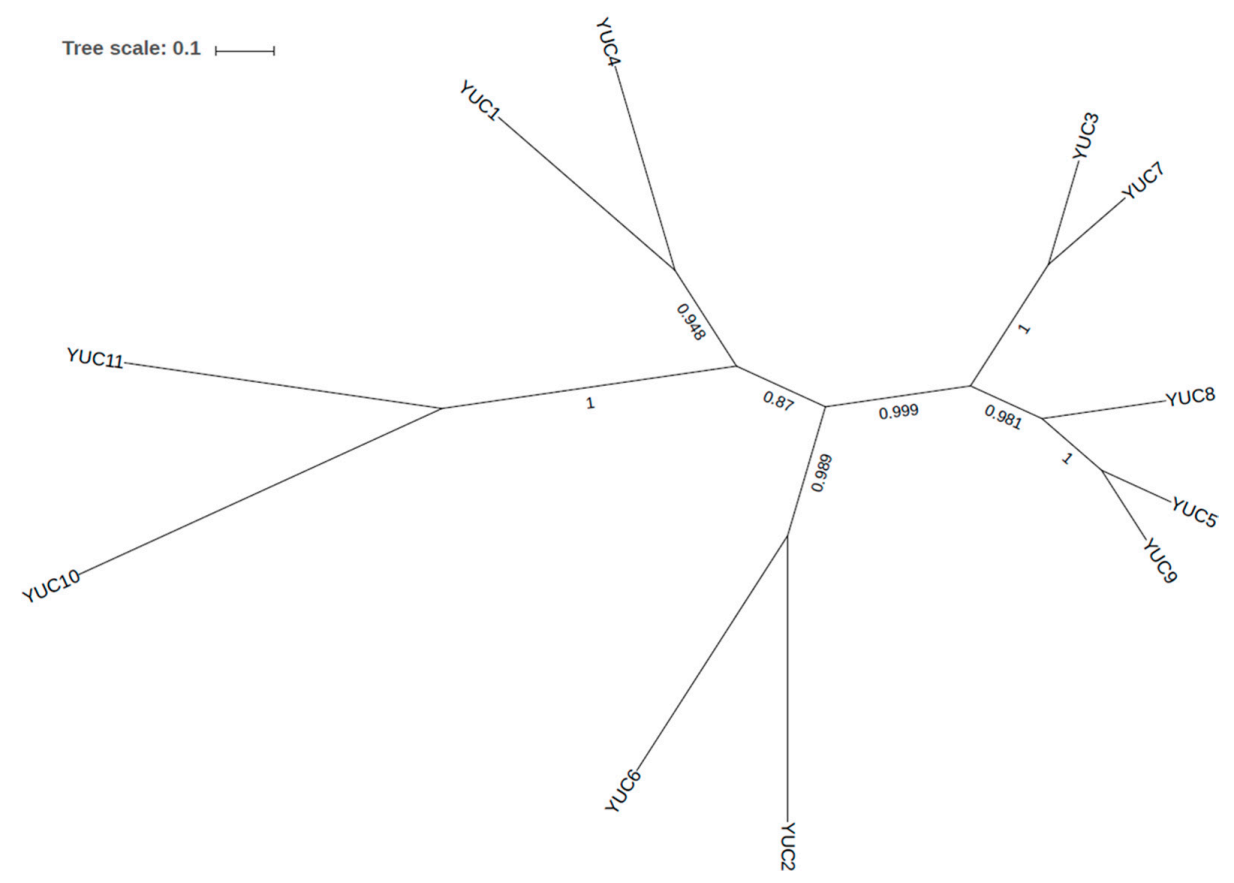

(B)

Figure 1. Phylogenetic tree of YUC proteins. Phylogenetic unrooted trees created using maximum likelihood methods on the proteins of interest resulting from an alignment are shown. Bootstrap values are given on the branches [16]. (A) Phylogenetic tree of all the homologues proteins detected for the 11 YUC proteins of arabidopsis. Bootstraps are only given in the branches, which split the different YUC clusters. Gene labels are constructed with the three letters from the species name (Table 1) and the gene name of the Arabidopsis thaliana homologues (Table S1); (B) Phylogenetic tree of the 11 YUC proteins detected in A. thaliana.

Table 1. Species for the phylogenetic analysis of the TAA/YUC auxin biosynthetic proteins. Species abbreviation are used in all the following phylogenetic and expression analyses.

\begin{tabular}{ccccc}
\hline Species & Abbreviation & Group & Genome & Chromosome No. \\
\hline Arabidopsis thaliana & Ath & eudicots & $150 \mathrm{Mb}$ & 5 \\
Arabidopsis lyrata & Aly & eudicots & $206.7 \mathrm{Mb}$ & 8 \\
Brassica rapa & Bra & eudicots & $283.8 \mathrm{Mb}$ & 10 \\
Prunus persica & $\mathrm{Ppe}$ & eudicots & $224.6 \mathrm{Mb}$ & 8 \\
Glycine max & $\mathrm{Gma}$ & eudicots & $950 \mathrm{Mb}$ & 20 \\
Carica papaya & $\mathrm{Cpa}$ & eudicots & $372 \mathrm{Mb}$ & 9 \\
Theobroma cacao & $\mathrm{Tca}$ & eudicots & $326.9 \mathrm{Mb}$ & 10 \\
Nelumbo nucifera & $\mathrm{Nnu}$ & eudicots & $804 \mathrm{Mb}$ & 8 \\
Vitis cinifera & $\mathrm{Vvi}$ & eudicots & $487 \mathrm{Mb}$ & 19 \\
Populus trichocarpa & $\mathrm{Ptr}$ & eudicots & $485 \mathrm{Mb}$ & 19 \\
Solanum lycopersicum & $\mathrm{Sly}$ & eudicots & $900 \mathrm{Mb}$ & 12 \\
Zea mays & Zma & monocots & $2.3 \mathrm{~Gb}$ & 10 \\
Oryza sativa & Osa & monocots & $372 \mathrm{Mb}$ & 12 \\
Musa acuminata & $\mathrm{Mac}$ & monocots & $472 \mathrm{Mb}$ & 8 \\
Amborella trichopoda & $\mathrm{Atr}$ & basal angiosperms & $706 \mathrm{Mb}$ & 13 \\
Pinus abies & $\mathrm{Pab}$ & gymnosperms & $19.6 \mathrm{~Gb}$ & 12 \\
Pinus lambertiana & $\mathrm{Pil}$ & gymnosperms & $31 \mathrm{~Gb}$ & 12 \\
Pinus taeda & $\mathrm{Pta}$ & gymnosperms & $20.15 \mathrm{~Gb}$ & 12 \\
Physcomitrella patens & $\mathrm{Ppa}$ & moss & $473 \mathrm{Mb}$ & 27 \\
Selaginella moellendorffii & Smo & lycophytes & $212.5 \mathrm{Mb}$ & 27 \\
\hline
\end{tabular}




\subsubsection{Phylogenetic Analysis for Shoot-Localised YUC Proteins}

For the shoot-localised YUC 1, 4, 2 and 6 proteins, 63 homologous proteins were detected ( 29 for YUC1-YUC4 group and 34 for YUC2-YUC6 group, Table S1). These proteins are only detected in the angiosperms (basal angiosperm, monocots and eudicots). YUC1 and YUC4 homologues are specific to brassicaceae; hence an event of gene duplication occurred specifically in brassicaceae (Figure S1) whereas YUC2-YUC6 gene duplication occurred after speciation between eudicots and monocots (Figure S2).

The majority of the proteins detected as homologous for YUC1-YUC4 and YUC2-YUC6 do not possess the predicted TMD features (Figures S1 and S2). For predicted membrane localisation, it is of interest that the earliest detected YUC1-YUC4 proteins in basal angiosperms both feature the predicted TMD (Figure S1). Later on, such predicted TMD can only be found in one out of eight monocot proteins (OsYUC1 4-b) and the two Nelumbo nucifera proteins, YUC1-a and YUC1-b. In the brassicaceae, TMD are predicted for BraYUC4-b and an alternate splice isoform of the arabidopsis YUC4 not annotated here [17]. Overall, this indicates that membrane localisation for the YUC1/YUC4 group was mostly lost over time.

The ancestral basal angiosperms show no indication of TMD in YUC2-YUC6 analysis and only a few proteins in monocots (banana MacYUC2 6-d), eudicots (cocoa tree TcaYUC2, soybean GmaYUC6-b, papaya CpaYUC6) and brassicaceae (field mustard BraYUC6-a) feature predicted TMD (Figure S2).

\subsubsection{Phylogenetic Analysis for Root-Localised YUC Proteins}

Sixty-five homologues were found in the YUC3-YUC7-YUC8-YUC5-YUC9 group; these proteins occur in gymnosperms and angiosperms, with no homologues detected in moss and club moss (Table S1). The majority of these proteins possess a predicted TMD on the N-terminus or in the middle of the protein, with the flavin-dependent monooxygenase domain constituting the rest of the protein.

One homologue of the YUC3-7-8-5-9 group was detected in basal angiosperms; eudicot homologues form two clusters, one for the YUC3-YUC7 homologues and the other one for YUC5-YUC8-YUC9. This suggests that YUC3-YUC7 and YUC5-YUC8-YUC9 were duplicated after the speciation between eudicots and monocots (Figure 2). The duplication of YUC3-YUC7 as well as the two gene duplications for YUC8 and YUC5-YUC9 are specific to brassicaceae. This group of proteins goes back to gymnosperms, and most proteins in this group feature a predicted TMD. In gymnosperms, only loblolly pine (PtaYUC3 578 9-c) and Norway spruce (PabYUC3 578 9-b) do not feature such a membrane anchor, but this is complemented by three or two other proteins, respectively, in Picea taeda and P. abies, that feature a predicted TMD. In the basal angiosperms, only amborella codes for a YUC3-5-7-8 protein (AtrYUC3 57 8) which also shows a TMD prediction. In monocots, all proteins in rice and maize feature TMD. In banana (Musa acuminata), with the largest number of proteins from the YUC3-7-8-5-9 group, five out of ten feature a predicted TMD. In eudicots, all but one protein in the YUC5-YUC8-YUC9 group feature predicted TMD, with poplar PtrYUC5-8-9 being the exception. The eudicot YUC3-YUC7 group also mainly features predicted TMD (Vitis vinifera, Prunus persica, Theobroma cacao) or at least one of two proteins contain sequences indicative of a TMD (P. trichocarpa, Glycine max). Tomato (Solanum lycopersicum) and papaya (Carica papaya) YUC3-YUC7 proteins do not feature TMD. In brassicaceae, only A. thaliana and A. lyrata YUC7 as well as one of the Brassica rapa YUC8 proteins (BraYUC8-b) do not feature a TMD indication. 


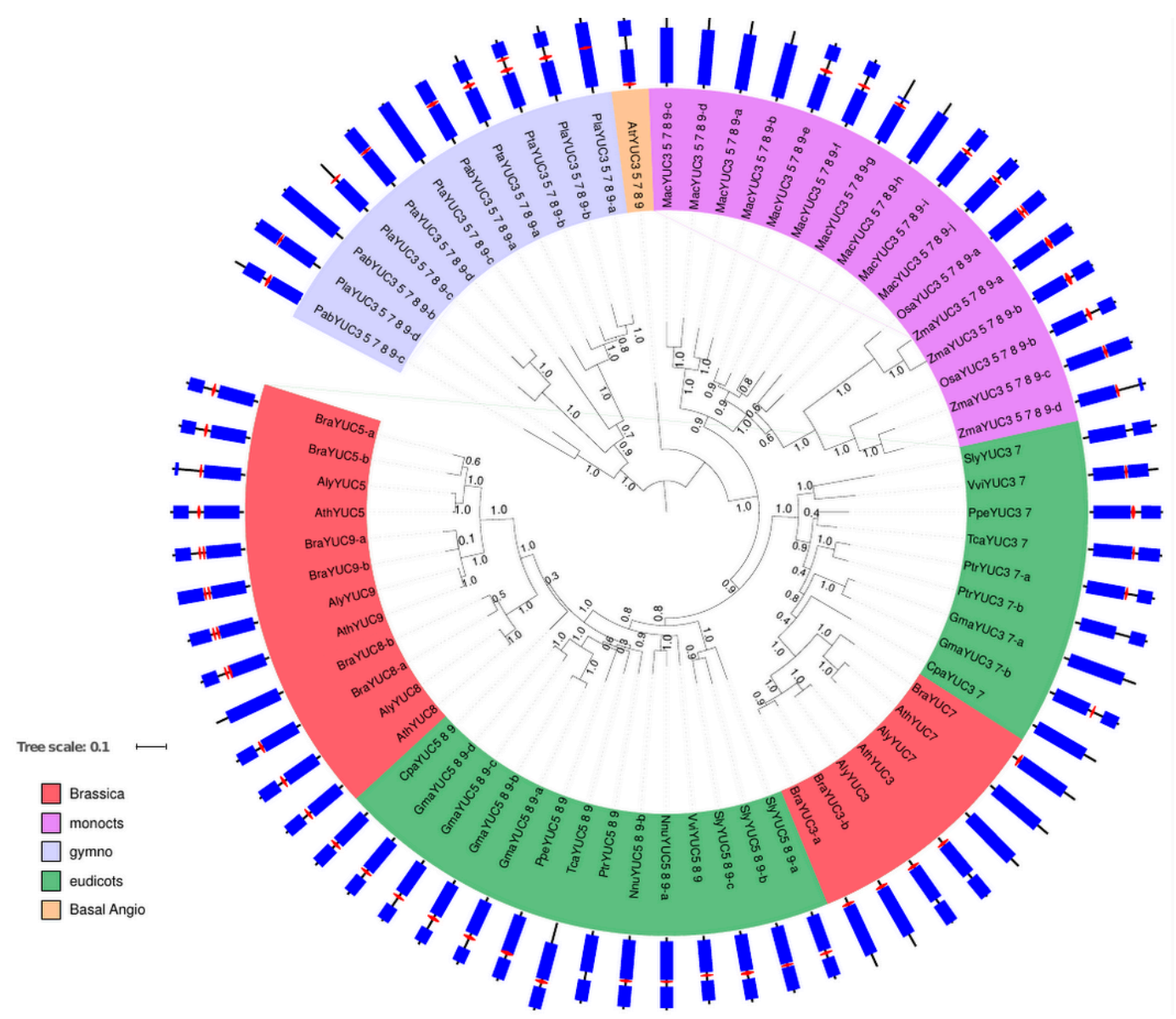

Figure 2. Phylogenetic tree of YUC3, YUC7, YUC8, YUC5 and YUC9 homologues proteins. A fast-tree using the maximum likelihood method [16] was applied on YUC3 YUC7 YUC8 YUC5 and YUC9 protein homologues constructed from an alignment using the MUSCLE (MUltiple Sequence Comparison by Log-Expectation) alignment tool with default parameters [18]. Bootstrap values are given on the branches. Gene labels are constructed with the three letters from the species name (Table 1) and the gene name of the $A$. thaliana homologues (Table S1). The color of the label shows the lineage of the plant. The corresponding protein domains (circle periphery) are obtained using InterProScan [15], red hexagons represent the TMD in the protein if applicable, and blue rectangles indicate the flavin-dependent monooxygenase domain typical for YUC proteins.

Overall, this is of interest as the YUC3-YUC7-YUC8-YUC5-YUC9 group is split in two groups from eudicots onwards: YUC3-YUC7 shows more of a tendency to cytosolic proteins and YUC5-YUC8-YUC9 to membrane-localised proteins. Throughout evolution, the proteins in the YUC5-YUC8-YUC9 group seem more consistent in featuring one or several predicted TMD than the YUC3-YUC7 group. This agrees with the arabidopsis data, where YUC5, 8, and 9 feature TMD but YUC7 does not, and YUC3 has a predicted TMD but still shows cytosolic localisation [8].

\subsubsection{Phylogenetic Analysis for YUC Proteins Localised in Flowers and Siliques}

In the YUC10-11 cluster, 59 proteins were found in all the species analysed. Therefore, these genes seem to be the most conserved in the YUC family and can be traced back to mosses and club mosses. In this group, the gene duplication between YUC10 and YUC11 occurred after the speciation between monocot and eudicots (Figure 3). The YUC10-YUC11 group features fewer TMD; e.g., in mosses and club mosses, only two proteins feature a predicted TMD. In gymnosperms, two Pinus taeda proteins (PtaYUC10 11-d and -e) and in basal angiosperms one amborella protein (AtrYUC10 11-c) potentially 
feature a TMD. Interestingly, in monocots, all banana and rice YUC10-YUC11 proteins have a TMD prediction but none of the maize proteins. Peach PpeYUC10 11 and cocoa TcaYUC10 11 show TMD predictions in the eudicots. None of the YUC10-YUC11 proteins in the brassicaceae analysed (B. rapa, A. thaliana and A. lyrata) show any TMD predictions (Figure 3).

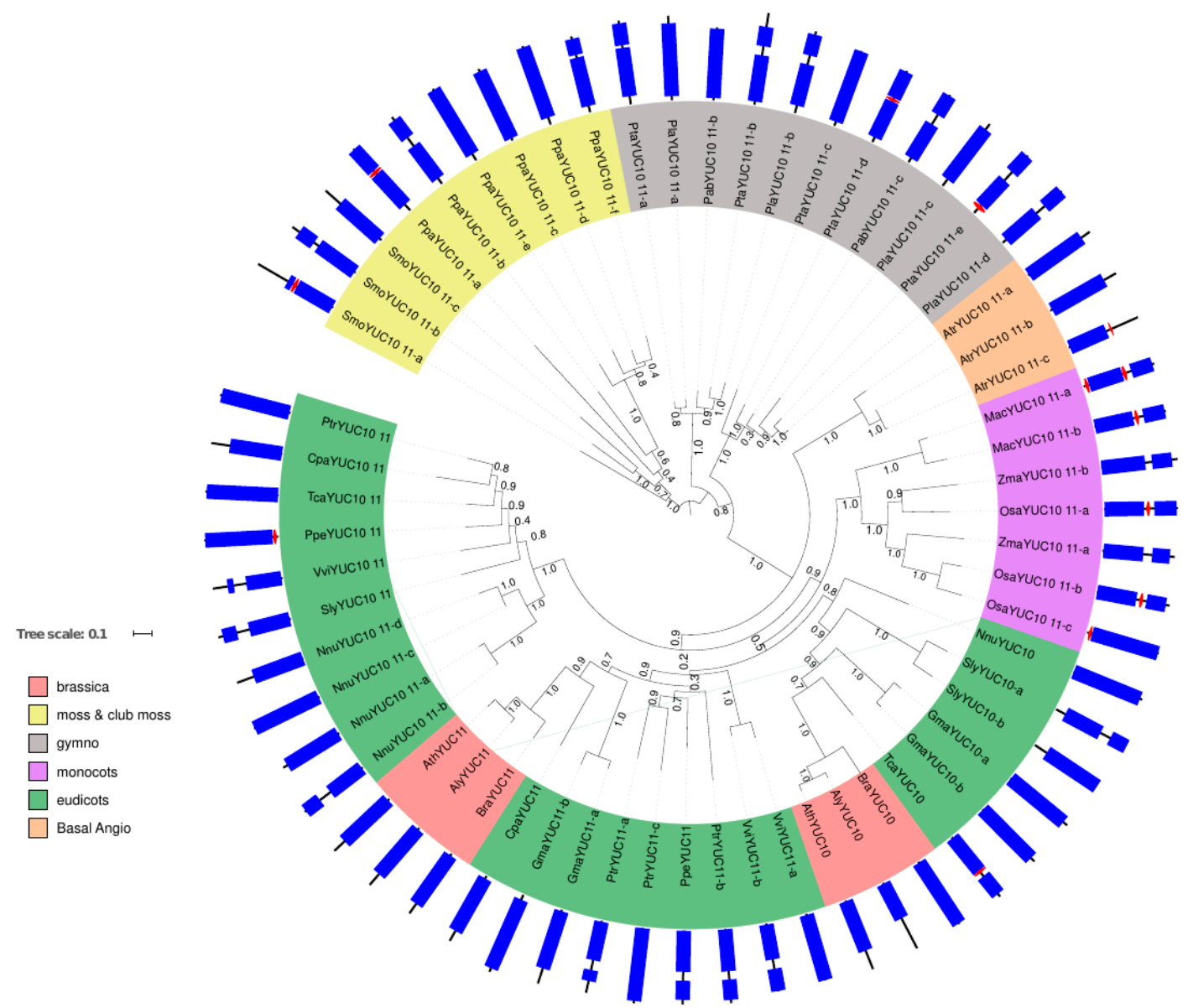

Figure 3. Phylogenetic tree of YUC10 and YUC11 homologous proteins. The tree is made using fast-tree with the maximum likelihood method [16] on YUC10 and YUC11 protein homologues constructed from an alignment using the MUSCLE alignment tool with default parameters [18]. Bootstrap values are indicated on the tree branches. Gene labels are constructed with the three letters from the species name (Table 1) and the gene name of the A. thaliana homologues (Table S1). The colour of the label shows the lineage of the plant. The corresponding protein domains (circle periphery) are obtained using InterProScan [15], red hexagons represent the TMD in the protein if applicable and blue rectangles indicate the flavin-dependent monooxygenase domain typical for YUC proteins.

In arabidopsis, AtYUC10 and AtYUC11 are suggested to have overlapping functions with AtYUC1 and AtYUC4 during embryogenesis [4]. All these proteins are cytosolic [8] except for the AtYUC4.2 splice variant which is flower specific [17]. Despite being seemingly the most ancient YUC isoforms, YUC10 and YUC11 seem to have adapted a specific role in embryogenesis in A. thaliana [4]. This is also represented in the RNAseq data for these genes below.

\subsection{Phylogenetic Analysis of TAR and TAA Proteins}

Three A. thaliana proteins were used as references to perform the phylogenetic analysis, or TAA/TAR proteins: AtTAR1, AtTAR2, and AtTAA1 (Table 2). These three proteins possess a tryptophan aminotransferase domain, but only TAR2 features a predicted TMD in the $\mathrm{N}$-terminal region. Due to 
the similarity in the homologues, a screen for the three proteins only one phylogenetic tree was created. The search with BLASTp and HMMER resulted in 57 proteins detected in all the species analysed (Table S2). The duplication of TAR1/TAA/TAR2 occurred during the speciation between monocots and eudicots. The eudicots clearly split into two groups: TAA1/TAR1 proteins featuring no predicted TMD and the TAR2 group (Figure 4). In these groups, most proteins indicate TMD, with the exceptions being one of the two lotus proteins NnuTAR2-a and one of two poplar proteins PtrTAR2-b. In brassicaceae, this division continues, but here all proteins in the TAR2 branch feature a predicted TMD and the cytosolic proteins are now divided into TAA1 and TAR1 proteins (Figure 4).

Table 2. Reference genes used in the study. The table summarizes information on the protein localisation in the plant and predicted protein domains (InterProScan).

\begin{tabular}{|c|c|c|c|c|c|c|}
\hline Gene Name & Gene ID & $\begin{array}{l}\text { Protein } \\
\text { Length }\end{array}$ & Localisation & $\begin{array}{c}\text { Flavin- } \\
\text { Dependent } \\
\text { Monooxygenase } \\
\text { Domain }\end{array}$ & $\begin{array}{c}\text { Tryptophan } \\
\text { Amino- } \\
\text { Transferase } \\
\text { Domain }\end{array}$ & $\begin{array}{c}\text { Predicted } \\
\text { TMD }\end{array}$ \\
\hline YUC1 & AT4G32540 & 414 & shoot + cytosolic & yes & no & No \\
\hline YUC2 & AT4G13260 & 415 & shoot + cytosolic & yes & no & No \\
\hline YUC3 & AT1G04610 & 437 & root + cytosolic & yes & no & Yes \\
\hline YUC5 & AT5G43890 & 424 & root $+\mathrm{ER}$ & yes & no & Yes \\
\hline YUC6 & AT5G25620 & 426 & shoot + cytosolic & yes & no & No \\
\hline YUC7 & AT2G33230 & 431 & root and ER & yes & no & No \\
\hline YUC8 & AT4G28720 & 426 & root + ER & yes & no & Yes \\
\hline YUC9 & AT1G04180 & 421 & root + ER & yes & no & Yes \\
\hline YUC10 & AT1G48910 & 383 & flower + cytosolic & yes & no & No \\
\hline TAR2 & AT4G24670 & 440 & whole plant and ER & no & yes & Yes \\
\hline
\end{tabular}

Following this duplication, TAR1 and TAA1 proteins show a high similarity. Both proteins are only found together in brassicaceae, so the duplication of these proteins occurred during brassicaceae speciation.

Regarding subcellular localisation throughout evolution for TAA/TAR proteins, the precursor proteins in mosses and club mosses are predicted to locate in the cytosol. In the gymnosperms, both Pinus lambertiana TAA/TAR proteins show TMD predictions, and two out of three Pinus taeda proteins do. Only Picea abies PabTAR TAA does not feature such a motif. In addition, the basal angiosperm amborella features a predicted TMD.

All in all, we are proposing that the phylogenetic groups detected also overlap with the biological function. YUC10-11 ancestry proteins are present in all species analysed, and therefore seem to be the oldest and potentially original proteins (Figure 5). During species diversification, their role seems to have become more specialised. In arabidopsis, AtYUC10 and AtYUC11 are suggested to have overlapping functions with AtYUC1 and AtYUC4 during embryogenesis [4]. A transcript for YUC10 is only detectable in flowers and siliques, and YUC11 is mostly transcribed in flowers (see RNAseq data below). In the YUC10-11 ancestral proteins, we have a mix of proteins with and without TMD predictions, with a lot of the monocot proteins featuring a predicted TMD, only two in eudicots and none in brassicaceae. YUC1-4 proteins occur for the first time in basal angiosperms, where they feature a TMD, whereas only one or two, respectively, TMD featuring YUC1/4 protein are detected in monocots and two representatives of the eudicots.

The next YUC group to develop is suggested to be YUC3-5-7-8-9, with over 95\% of the proteins featuring predicted TMD; YUC3-5-7-8-9 then splits into two groups in eudicots, YUC3-YUC7 and YUC5-8-9 (Figure 5). Still, most of the proteins derived from these groups contain predicted TMD with YUC7 proteins being more likely not to carry a TMD. 


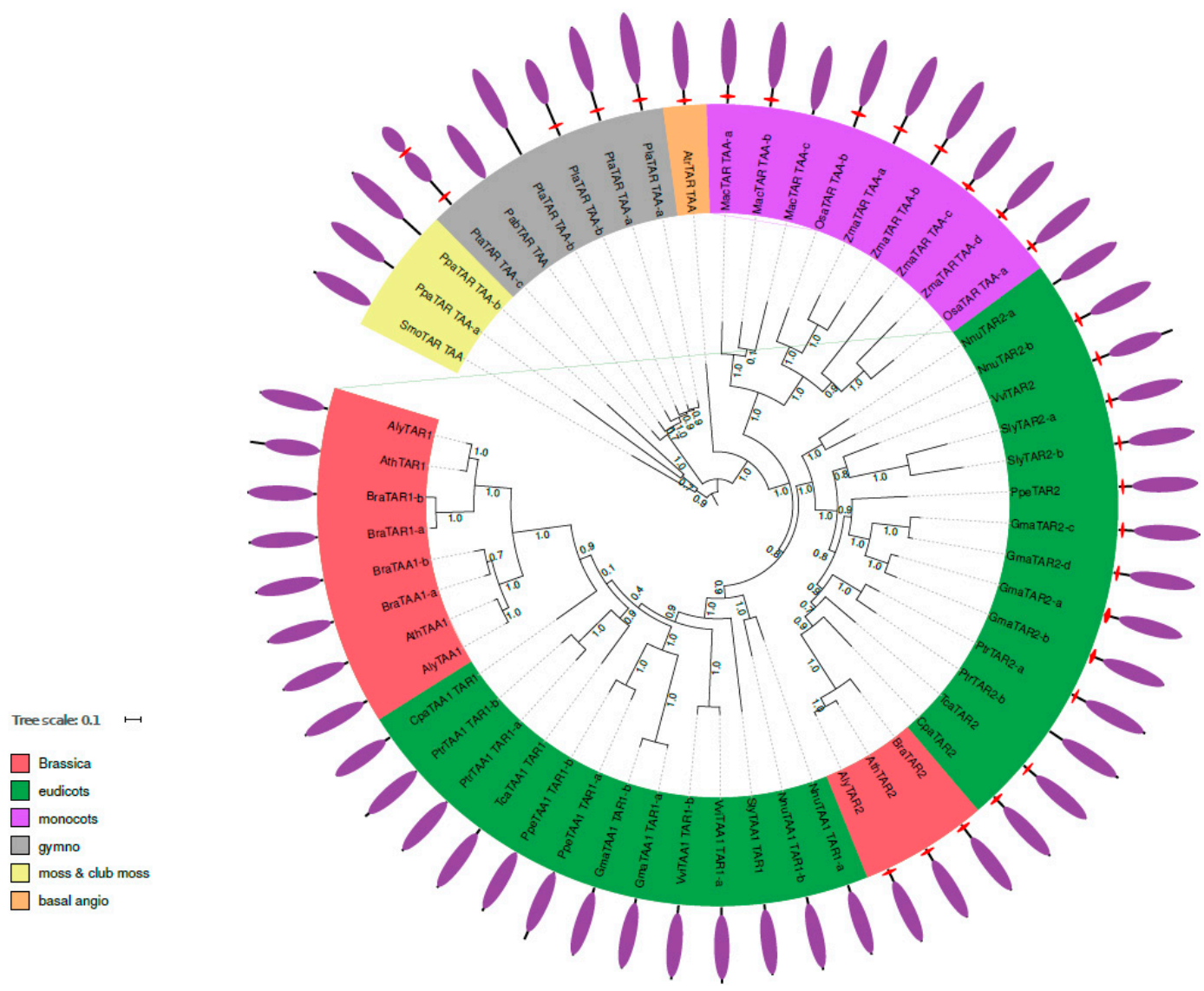

Figure 4. Phylogenetic tree of TAR1, TAR2 and TAA1 homologous proteins. Fast-tree using the maximum likelihood method [16] was used to create the phylogenetic tree for TAR1, TAR2 and TAA1 protein homologues constructed from MUSCLE alignment [18]. Bootstrap values are indicated on the branches of the phylogenetic tree. Gene labels are constructed with the three letters from the species name (Table 1) and the gene name of the A. thaliana homologue (Table S1). The colour of the label shows the lineage of the plant. The corresponding protein domains (circle periphery) are obtained using InterProScan [15], red hexagons represent the TMD in the protein if applicable and purple ellipses represent tryptophan aminotransferase domain.

Original TAA/TAR proteins do not feature predicted TMD; the first TMD prediction can be detected in gymnosperms and basal angiosperms. Interestingly, similar to YUC1 and YUC4 proteins, TAA and TAR proteins do not divide into two groups in monocots but do in eudicots. However, whereas YUC1 and YUC4 proteins mainly do not show TMD predictions in both proteins, the TAA/TAR groups splits into TAA and TAR1 proteins without, and, in the case of TAR proteins, mainly with, a TMD.

In this context, it is of interest that TAR1 and TAR2 single mutant displays no visible phenotype and TAA1 show altered responses to shade and a weak ethylene insensitivity that can be rescued by IAA but no developmental defects under normal growth conditions [5]. The TAA1/TAR2 double mutants have defects in their vascular and floral development and are sterile [5]. Triple mutants (TAA1/TAR1/TAR2) lack a primary root and visible vasculature in their cotyledons, have a reduced hypocotyl, and are more likely to develop a single cotyledon than wild-type embryos [5]. Other than YUC proteins that show defects in root or shoot, respectively, depending on whether the mutations are in ER-localised or cytosolic YUC proteins [6], TAA and TAR proteins seem to have overlapping functions in root and shoot tissue, which is also reflected in their RNAseq profile and will be discussed in the next paragraph. 


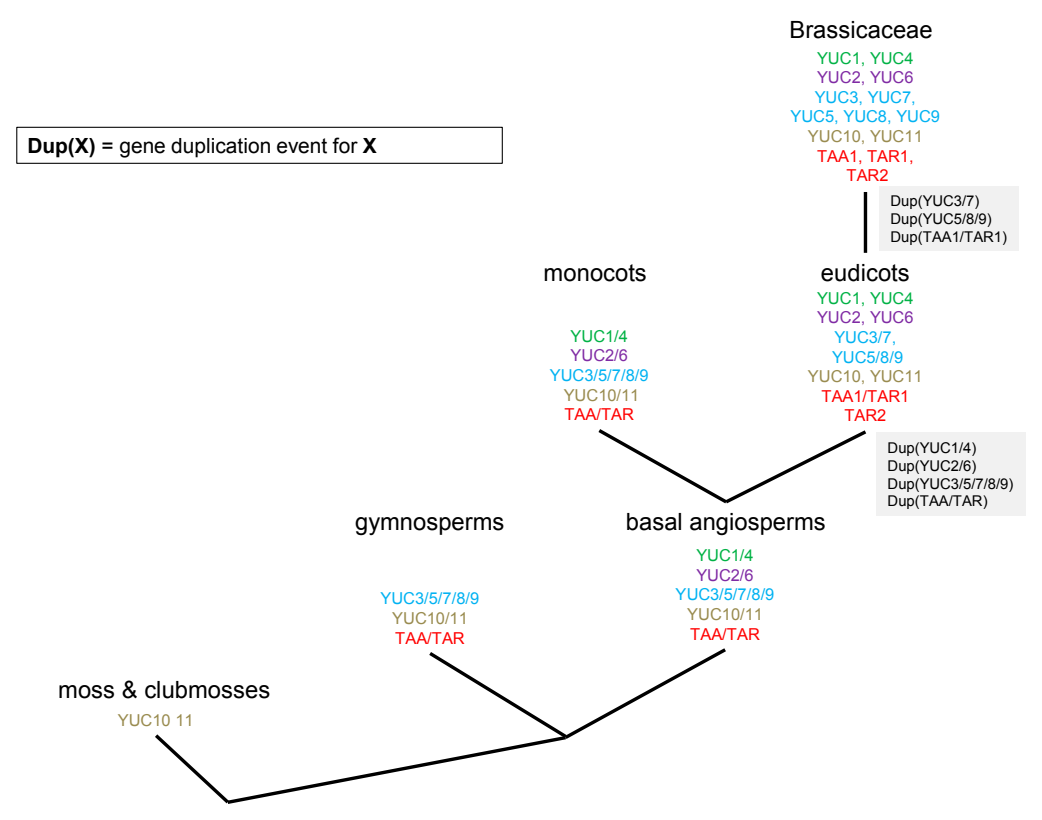

Figure 5. Evolutionary development of auxin biosynthetic enzymes in the TAA/YUC pathway. Nodes show an event of speciation, which is correlated with the appearance or the duplication of YUC or TAR/YUC genes. The name gene colors indicate the different "gene families".

\subsection{RNAseq Analysis of Auxin Biosynthetic Enzymes in the TAA/YUC Pathway}

In arabidopsis, auxin biosynthetic activity can be found associated with both cytosolic as well as ER-membrane fractions [8], and a subset of auxin biosynthetic proteins show ER-localisation, whereas other enzymes localize in the cytosol [8]. Mutant studies suggest YUC1, 2, 4 and 6 to be the main YUCs in shoots and YUC3, 5, 7, 8 and 9 in roots [6].

To analyse the tissue distribution of auxin biosynthetic enzymes, an RNAseq analysis for TAA1, TAR1, TAR2 as well as the 11 YUC transcripts was carried out (Figure 6 and Tables S1-S3). Datasets were obtained for the following tissues: root meristems, primary roots, cotyledons, 14-day-old seedlings, adult leaves, flowers, siliques, and floral buds. The data available on NCBI (https:/ / www.ncbi.nlm. nih.gov/geo) were mapped against The Arabidopsis Information Resource (TAIR) database, and then the reads per kilobase of transcript per million mapped reads (RPKM) was computed for all genes of interest and normalised (see Material and Methods).

It is interesting to note that, for the first step of auxin biosynthesis, TAA1 is far more predominant than TAR2. Only in siliques can we find higher transcript levels for TAR2 than for TAA1 and approximately equal levels in flowers, as well as in seedlings and adult leaves. TAR 1 is only present in flowers and siliques at very low transcript levels (Figure 6).

For the YUC genes and the second step in YUC-dependent auxin biosynthesis, YUC3 appears to be the predominant transcript in the root meristem and primary root. YUC3 is an interesting protein, as phylogenetically it groups with the ER-membrane bound YUC proteins and shows predictions for a TMD but shows cytosolic localization [8]. The ER-localised YUCs (YUC5, 7, 8, and 9) have their highest transcript levels in root meristem and primary root tissue. YUC5, 8 and 9 have a high level of amino acid identity $(\sim 75 \%)$ and are most likely the result of gene duplications occurring between the evolution of the eudicots and brassicaceae. YUC5 is mainly transcribed in primary roots. YUC8 transcript levels decrease from rot meristem over primary root to cotyledons, whereas YUC9 is at its highest level in primary root tissue. YUC7 is mainly transcribed in the root meristem (Figure 6).

In the cytosolic group, YUC1—probably the most studied YUC enzyme-and YUC4 are not detected in root meristem and primary root and are transcribed at very low levels otherwise, with a slight increase in flowers and floral buds. YUC6 is transcribed throughout the tissues analysed, with 
slightly higher transcription levels in primary roots and lower levels in adult leaves. YUC10 is only detectable in flowers and siliques at very low levels; the last cytosolic protein YUC11 is transcribed throughout at low levels with a slight maximum in flowers.

RNA sequencing transcript analysis was carried out for TAA1, TAR1, TAR2 and YUC1, 2, 3, 4, 5, $6,7,8,9,10,11$ in root meristem, primary root, cotyledon, seedling 14 days after germination, adult leaves, flower, silique and floral bud tissue (Table 3 and see Material and Methods section). Data is given as RPKM (reads per kilobase of transcript per million mapped reads) and was normalised to the reference gene AtSAND (At2g28390).

Table 3. Sequence Read Archives (SRA) files used for RNA sequencing analysis. All SRA files were downloaded from http:/ / www.ncbi.nlm.nih.gov/geo/browse/.

\begin{tabular}{cc}
\hline Tissue & SRA File Number for RNAseq Analysis \\
\hline \multirow{3}{*}{ Root meristematic zone } & SRR1740401 \\
& SRR1740402 \\
SRR1740403 \\
\hline \multirow{2}{*}{ Primary root } & SRR1042766 \\
& SRR1042767 \\
\hline \multirow{2}{*}{ Cotyledon } & SRR1292207 \\
& SRR605657 \\
Seedling 14 dag & SRR605658 \\
& SRR346552 \\
& SRR346553 \\
\hline Adult Leaves & SRR1159821 \\
& SRR1159827 \\
& SRR1159831 \\
\hline Flower & SRR1159837 \\
\hline Silique & SRR656217 \\
\hline \multirow{2}{*}{ Flower bud } & SRR656218 \\
\hline
\end{tabular}

To look into the distribution of proteins with and without the predicted TMD in the different tissues, and especially in terms of the mutant data [6] in root and shoot tissues, transcript levels for YUC1, 2, 4, 6, 10, and 11 and transcript levels for YUC3, 5, 7, 8, and 9 were compiled for each tissue (Figure 7).

For TAA/TAR, the cytosolic TAA1 and TAR1 which do not feature a predicted TMD show equal or higher transcript levels than the ER-membrane bound TAR2 with a predicted TMD, with the exception of silique tissue, where TAR2 is the main transcript (Figure 7A). For the YUC genes, the cytosolic YUCs are the main transcripts in seedlings, flower, silique and flower bud (Figure 7B). The main transcripts in root meristem and primary root are indeed the ER-membrane bound YUCs, together with YUC3 (Figure 7B). YUC3 does feature a predicted TMD but has not shown ER-localisation in plants [8]. YUC3 indeed accounts for the majority of transcript in root meristem tissue (Figure 6). These findings align very well with mutant data: YUC1-2-4-6 quadruple mutants have severe defects in vascular patterning and flower development but have no root defects, which is consistent with the expression patterns [6]. Quintuple mutants of YUC3, 5, 7, 8 and 9 (yucQ mutant) develop short and agravitropic roots [6]. The $y u c Q$ phenotypes are rescued by IAA addition or by expressing a YUC gene in yucQ roots but not by overexpression of a YUC gene in shoots [6]. The root phenotypes of the yucQ mutant (yuc3/5/7/8/9) can be rescued by YUC overexpression in the mutant roots but not in shoots [6], highlighting the necessity of local auxin biosynthesis. Furthermore, the phylogenetic class of YUC3, 5, 7, 8, and 9 seems to be mainly involved in auxin biosynthesis in the roots, while the class containing the cytosolic proteins YUC1, 2, 4, 6, 10, and 11 might be more important for auxin biosynthesis in shoots, with YUC10 and 11 only present in flowers and siliques. 


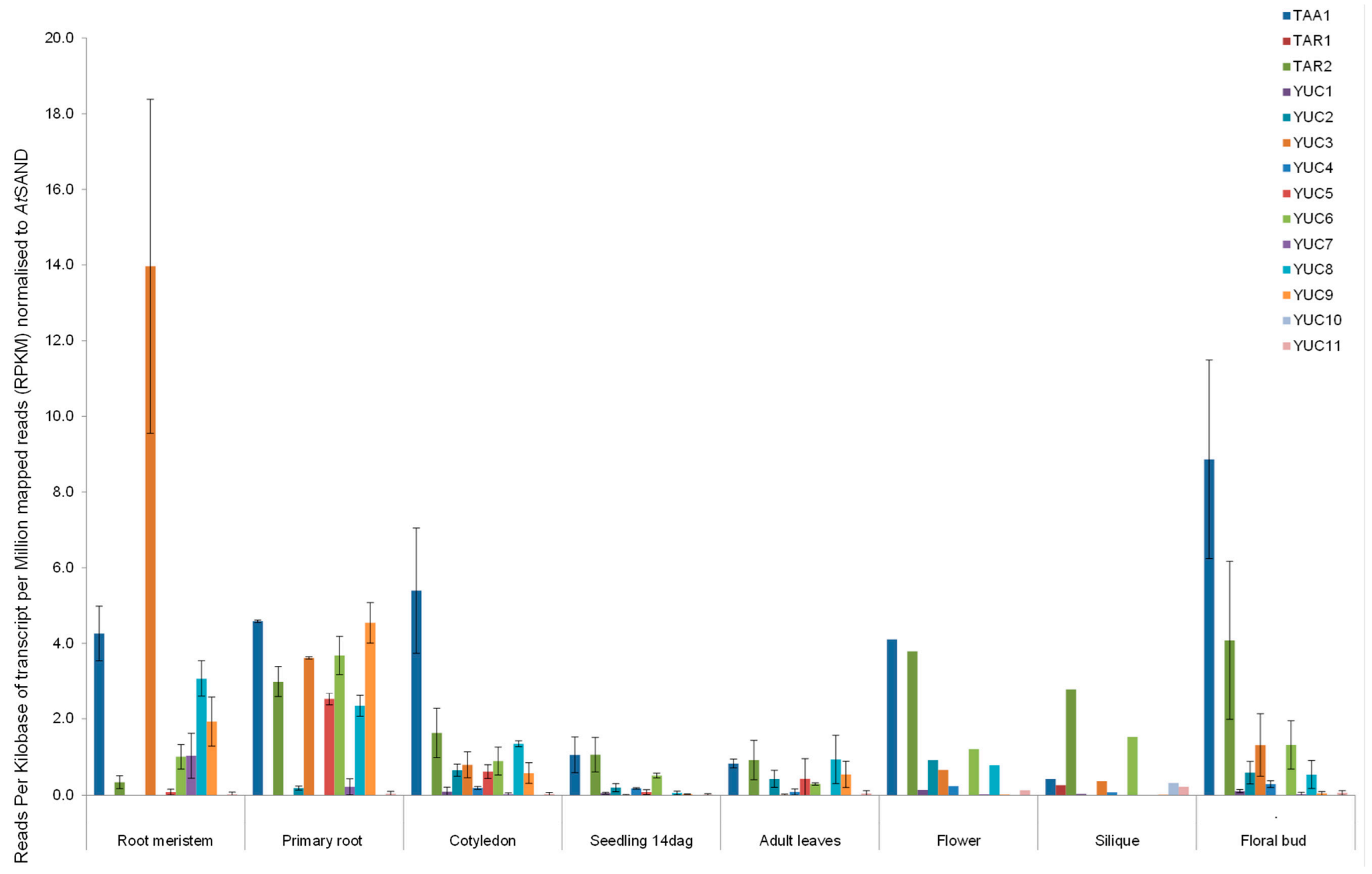

Figure 6. Tissue-specific distribution of TAA/TAR and YUC transcripts. 


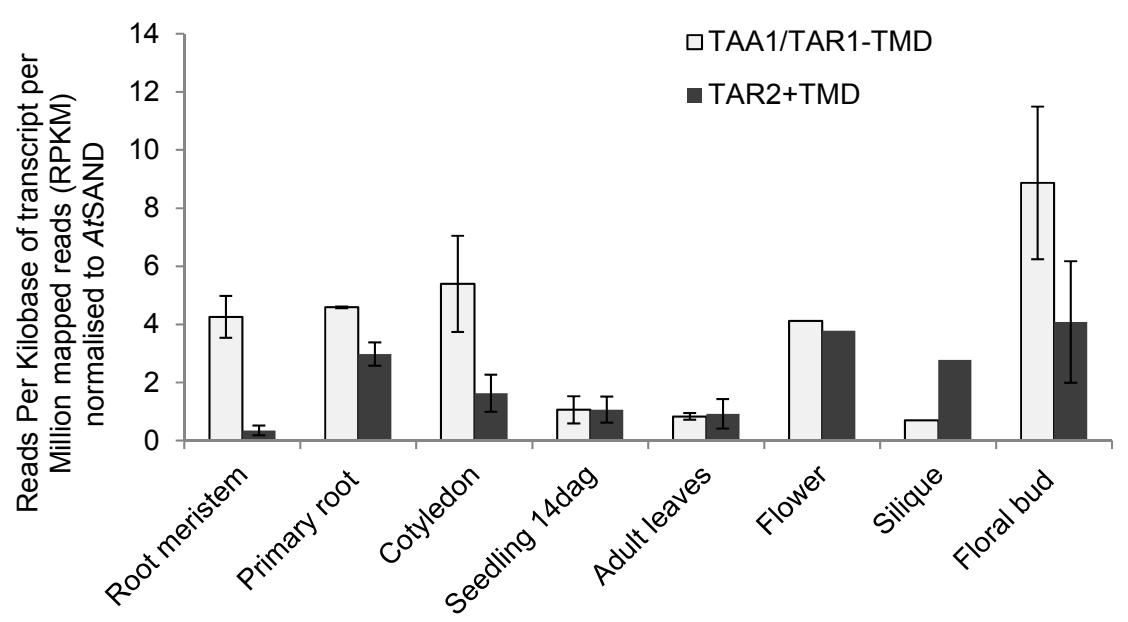

(A)

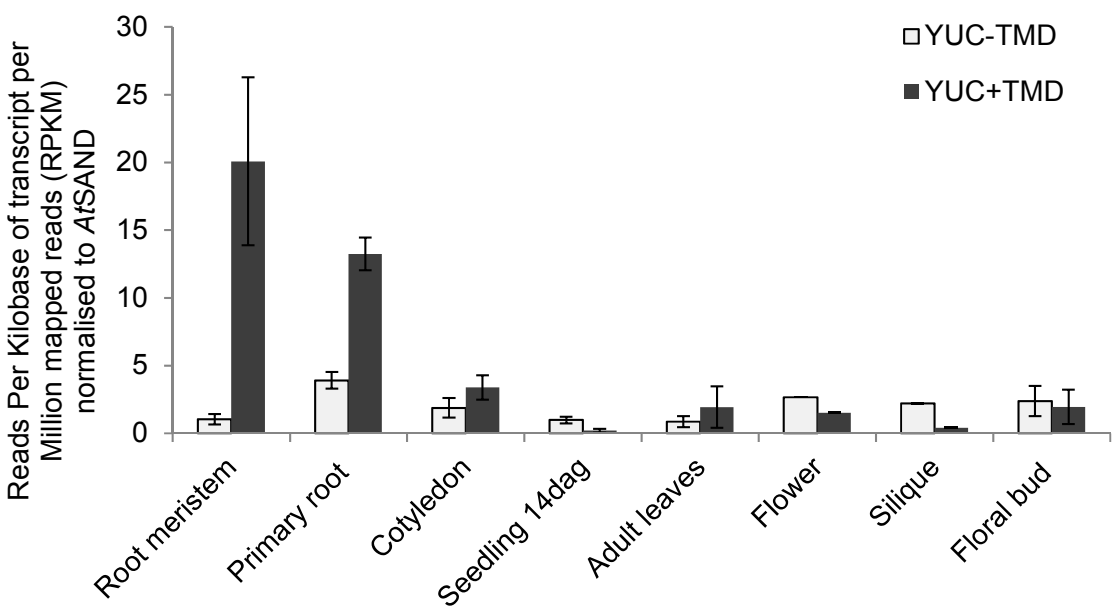

(B)

Figure 7. Tissue-specific distribution of TAA/TAR and YUC transcripts with and without predicted TMD. (A) RNA sequencing transcript analysis for TAA/TAR proteins without TMD (TAA1, TAR1) and with TMD prediction (TAR2) in root meristem, primary root, cotyledon, seedling 14 dag, adult leaves, flower, silique and floral bud tissue (Table 3 and see Material and Methods section). Data is given as RPKM (reads per kilobase of transcript per million mapped reads) and was normalised to the reference gene AtSAND (At2g28390); (B) RNAseq transcript analysis for YUC proteins without TMD (YUC1, 2, 4, $6,10,11)$ and with TMD prediction (YUC3, 5, 7, 8, 9) in root meristem, primary root, cotyledon, seedling $14 \mathrm{dag}$, adult leaves, flower, silique and floral bud tissue (Table 3 and see Material and Methods section) Data is given as RPKM (reads per kilobase of transcript per million mapped reads) and was normalised to the reference gene AtSAND (At2g28390).

\section{Material and Methods}

\subsection{Homologous Protein Detection}

For all proteins of interest, a basic local alignment search tool (BLASTp, NCBI, Bethesda, MD, USA) was used with default parameters as well as the hidden Markov model-based sequence alignment tool (HMMER, EMBL-EB, Hinxton, UK, http//hmmer.org). Best hits were retained and used for phylogenetic analysis [19]. The proteome of each species was used as reference for the two analyses (Table 1), and the YUC, TAR and TAA proteins of Arabidopsis were used as queries (Table 2). Analysis results are shown in Table S1 for YUC proteins and Table S2 for TAR/TAA 
proteins. InterProScan (EMBL-EB, Hinxton, UK) [15] was applied for domain predictions (TMD, flavin-dependent monooxygenase domains, tryptophan aminotransferase domains). InterProScan (EMBL-EB, Hinxton, UK) is a platform that integrates multiple and diverse databases for the domain prediction allowing for a comparative but integrated approach whilst reducing redundancy and capitalizing on the individual strengths of the different databases. Reciprocal BLASTp (NCBI, Bethesda, MD, USA) and domain analysis with InterProScan (EMBL-EB, Hinxton, UK) [15] were used to verify the relevance of all identified orthologues. The protein data used in this analysis were downloaded from three different databases: plaza genomic (http://bioinformatics.psb.ugent.be/plaza), phytozome (https:/ / phytozome.jgi.doe.gov/pz/portal.html) and congenie (http:// congenie.org/); nomenclature was used according to these databases.

\subsection{Phylogenetic Reconstruction}

Selected sequences were first aligned with MUSCLE (EMBL-EB, Hinxton, UK), a multiple sequence alignment tool [18], using default parameters. For the construction of the phylogenetic tree Fast-Tree was applied using the default parameters [16]. Fast-Tree infers approximately-maximum-likelihood phylogenetic trees from alignments. Finally, phylogenetic trees were drawn using the Interactive Tree Of Life ITOL (EMBL, Heidelberg, Germany) [20]. The protein domains shown in the different phylogenetic clades are detected with INTERPROSCAN (EMBL-EB, Hinxton, UK) [15]

\subsection{RNA Sequencing Data and Analysis}

Data used for the RNAseq analysis was obtained from the NCBI (http:/ / www.ncbi.nlm.nih.gov / geo/browse/). Five different tissues (leaves, roots, flowers, flower buds, and seeds/siliques) as well as total seedling were chosen for the analysis of the expression patterns of the genes of interest in A. thaliana (Table 3). Reads from RNAseq libraries were mapped onto the candidate gene sequences allowing no mismatches using TOPHAT v 2.0.14 (University of Maryland, MD, USA) [21] with standard settings and maximum of multihits set at 1 , minimum intron length set at $15 \mathrm{bp}$, and maximum intron length set as $6000 \mathrm{bp}$. Reads were added together for each gene using HTseq-count with the overlap resolution mode set as intersection-non empty and with no strand-specific protocol [22]. Transcription levels in reads per kilobase of transcript per million mapped reads (RPKM) were normalised to AtSAND (At2g28390). This gene was chosen due to its constant gene expression levels across different tissues at developmental stages in A. thaliana [23].

\subsection{Confocal Imaging of Arabidopsis Seedlings}

A. thaliana seeds expressing GFP-HDEL in a stable manner were sterilized with $10 \%(v / v)$ bleach and then rinsed in sterile distilled, deionized water. The seeds were plated in petri dishes on Murashige and Skoog medium with $1 \%(w / v)$ sucrose, solidified with $1.2 \%(w / v)$ phytoagar, and grown in 16-h photoperiods at $22{ }^{\circ} \mathrm{C}$ for 7 days. Images were taken using a Zeiss 880 laser scanning confocal microscope (Zeiss, Oberkochen, Germany) with $\times 100 / 1.46$ NA oil immersion objective. For imaging of the GFP samples were excited using the 488 laser line with detection in the 494- to 513-nm range. Images were edited using the ZEN image browser (Zeiss, Oberkochen, Germany).

\section{Conclusions}

We hypothesize that membrane anchoring via TMD to the endoplasmic reticulum in TAA/YUC auxin biosynthesis could already be found early on in evolution in mosses and club mosses. The distribution between the different phylogenetic classes in root and shoot may well originate from gene duplications. The original YUC10-11 proteins then adapted more specialized functions in flowers and siliques, together with cytosolic YUC1-YUC4 proteins. The phylogenetic group of YUC5,8,9 features predicted TMD throughout evolution and in arabidopsis can mainly be found and is active in root tissue. This might be linked to the high abundance of ER in arabidopsis roots compared 
to leaf tissue (Figure 8) and a difference in network structure as well as functional requirements of this tissue such as enhanced secretion. ER-linked auxin biosynthesis might therefore be important for a variety of plant species throughout evolution. So far, this has been tested in maize and arabidopsis. We have shown that, for maize coleoptiles and primary root [7] and arabidopsis whole seedlings [8], about a quarter of auxin biosynthetic activity can be found in microsomal fractions containing ER membranes. In the light of this phylogenetic and transcription data, it would therefore be of great interest to test the contribution of ER-localised and cytosolic auxin biosynthesis in different species throughout evolution and link it to the abundance of the corresponding proteins, with and without TMD. Narrowing down such analysis to different tissues, e.g., root and shoot in arabidopsis, also has the potential to reveal a difference in regulation of TAA/YUC auxin biosynthesis, which might be dependent on tissue function and subcellular structures etc.
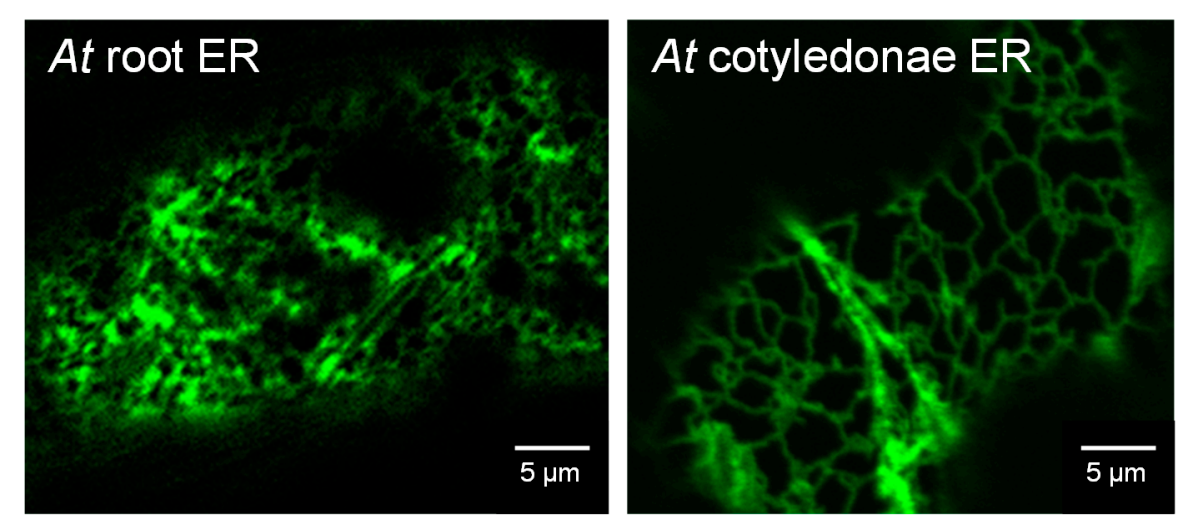

Figure 8. Structure of the endoplasmic reticulum (ER) in arabidopsis root and cotyledonae. Confocal images of ER in arabidopsis root and cotyledonae seven days after germination. Arabidopsis transformed in a stable manner, with the ER lumenal marker GFP-HDEL (green fluorescent protein fused to an ER retention signal consisting of the four amino acids Histidine-Aspartic acid-Glutamine-Leucine) used to visualize the ER.

For arabidopsis and the transcriptional data, it will be of great interest to further elucidate the role and contribution of YUC3 to auxin biosynthesis due to the high abundance of the transcript especially in the root meristem. It is also interesting that enzymes in the two steps of TAA/YUC-dependent auxin biosynthesis display differences in the tissue distribution between proteins with and without TMD: for the first step, TAA1 and TAR2 are the main forms, with the cytosolic TAA1 being more predominant than the ER-localised TAR2. TAR2 shows higher transcript levels than TAA1 only in siliques. In contrast to that, YUC proteins with predicted TMD show higher transcript levels in root tissues whereas proteins without predicted TMD seem to be higher transcribed in leaf and flower tissues (Figure 7). Enzymatic steps in one pathway should be more efficient if the enzymes are in proximity, or at least in the same subcellular compartment, if transport of intermediates is to be avoided. It is therefore curious that, for the first step in the biosynthesis, a cytosolic TAA1 is predominant, whereas for the second step, the YUC proteins show tissue specificity between TMD and non-TMD proteins. This could suggest protein-protein interactions and metabolon formation as suggested before for auxin biosynthesis [8] but detailed proteomics and localisation analysis including membrane topology are necessary to shed light on this matter.

All in all data, derived from phylogenetic analysis and arabidopsis transcription data fits well with mutant studies [6], indicating a subgroup of YUC proteins that are localized to the ER and seem predominant in root tissues. Already in the simple basal angiosperm, such as amborella, which underwent only few whole genome duplications, TAA/YUC-dependent auxin biosynthesis is already complex and displays differentiation in ER-membrane bound and cytosolic proteins. 
This could also indicate the conservation of differences for auxin biosynthesis in root and leaf since the basal angiosperms.

Supplementary Materials: Supplementary materials can be found at www.mdpi.com/1422-0067/18/8/1791/s1. Acknowledgments: The authors thank David Evans for critical reading of the manuscript.

Author Contributions: Axel Poulet carried out the phylogenetic analysis and gathered the RNAseq data. Verena Kriechbaumer designed the project and analysed the RNAseq data. Both authors wrote the manuscript.

Conflicts of Interest: The authors declare no conflict of interest.

\section{References}

1. Zhao, Y. Arabidopsis Book; The American Society of Plant Biologists: Rockville, MD, USA, 2014; Volume 12.

2. Tivendale, N.D.; Ross, J.J.; Cohen, J.D. The shifting paradigm of auxin biosynthesis. Trends Plant Sci. 2014, 19, 44-51. [CrossRef] [PubMed]

3. Zhao, Y.; Christensen, S.K.; Fankhauser, C.; Cashman, J.R.; Cohen, J.D.; Weigel, D.; Chory, J. A role for flavin monooxygenase-like enzymes in auxin biosynthesis. Science 2001, 291, 306-309. [CrossRef] [PubMed]

4. Cheng, Y.; Dai, X.; Zhao, Y. Auxin biosynthesis by the YUCCA Flavin monooxygenases controls the formation of floral organs and vascular tissues in Arabidopsis. Genes Dev. 2006, 20, 1790-1799. [CrossRef] [PubMed]

5. Stepanova, A.N.; Robertson-Hoyt, J.; Yun, J.; Benavente, L.M.; Xie, D.Y.; Dolezal, K.; Schlereth, A.; Jürgens, G.; Alonso, J.M. TAA1-mediated auxin biosynthesis is essential for hormone crosstalk and plant development. Cell 2008, 133, 177-191. [CrossRef] [PubMed]

6. Chen, Q.; Dai, X.; De-Paoli, H.; Cheng, Y.; Takebayashi, Y.; Kasahara, H.; Kamiya, Y.; Zhao, Y. Auxin overproduction in shoots cannot rescue auxin deficiencies in Arabidopsis roots. Plant Cell Physiol. 2014, 55, 1072-1079. [CrossRef] [PubMed]

7. Kriechbaumer, V.; Seo, H.; Park, W.J.; Hawes, C. Endoplasmic reticulum localization and activity of maize auxin biosynthetic enzymes. Plant Physiol. 2015, 169, 1933-1945. [CrossRef] [PubMed]

8. Kriechbaumer, V.; Botchway, S.W.; Hawes, C. Localization and interactions between Arabidopsis auxin biosynthetic enzymes in the TAA/YUC-dependent pathway. J. Exp. Bot. 2016, 67, 4195-4207. [CrossRef] [PubMed]

9. Tao, Y.; Ferrer, J.L.; Ljung, K.; Pojer, F.; Hong, F.; Long, J.A.; Li, L.; Moreno, J.E.; Bowman, M.E.; Ivans, L.J.; et al. Rapid synthesis of auxin via a new tryptophan-dependent pathway is required for shade avoidance in plants. Cell 2008, 133, 164-176. [CrossRef] [PubMed]

10. Mravec, J.; Skupa, P.; Bailly, A.; Hoyerová, K.; Krecek, P.; Bielach, A.; Petrásek, J.; Zhang, J.; Gaykova, V.; Stierhof, Y.D.; et al. Subcellular homeostasis of phytohormone auxin is mediated by the ER localized PIN5 transporter. Nature 2009, 459, 1136-1140. [CrossRef] [PubMed]

11. Dal Bosco, C.D.; Dovzhenko, A.; Liu, X.; Woerner, N.; Rensch, T.; Eismann, M.; Eimer, S.; Hegermann, J.; Paponov, I.A.; Ruperti, B.; et al. The endoplasmic reticulum localized PIN8 is a pollen-specific auxin carrier involved in intracellular auxin homeostasis. Plant J. 2012, 71, 860-870. [CrossRef] [PubMed]

12. Ding, Z.; Wang, B.; Moreno, I.; Dupláková, N.; Simon, S.; Carraro, N.; Reemmer, J.; Pěnčík, A.; Chen, X.; Tejos, R.; et al. ER-localized auxin transporter PIN8 regulates auxin homeostasis and male gametophyte development in Arabidopsis. Nat. Commun. 2012, 3, 941. [CrossRef] [PubMed]

13. Barbez, E.; Kubes, M.; Rolcik, J.; Béziat, C.; Pěnčík, A.; Wang, B.; Rosquete, M.R.; Zhu, J.; Dobrev, P.I.; Lee, Y.; et al. A novel putative auxin carrier family regulates intracellular auxin homeostasis in plants. Nature 2012, 485, 119-122. [CrossRef] [PubMed]

14. Bender, R.L.; Fekete, M.L.; Klinkenberg, P.M.; Hampton, M.; Bauer, B.; Malecha, M.; Lindgren, K.; A Maki, J.; Perera, M.A.; Nikolau, B.J.; et al. PIN6 is required for nectary auxin response and short stamen development. Plant J. 2013, 74, 893-904. [CrossRef] [PubMed]

15. Finn, R.D.; Attwood, T.K.; Babbitt, P.C.; Bateman, A.; Bork, P.; Bridge, A.J.; Chang, H.-C.; Dosztányi, Z.; El-Gebali, S.; Fraser, M.; et al. InterPro in 2017-beyond protein family and domain annotations. Nucleic Acids Res. 2017, 45, D190-D199. [CrossRef] [PubMed]

16. Price, M.N.; Dehal, P.S.; Arkin, A.P. Fasttree 2 approximately maximum-likelihood trees for large alignments. PLoS ONE 2010, 5, e9490. [CrossRef] [PubMed] 
17. Kriechbaumer, V.; Wang, P.; Hawes, C.; Abell, B.M. Alternative splicing of the auxin biosynthesis gene YUCCA4 determines its subcellular compartmentation. Plant J. 2012, 70, 292-302. [CrossRef] [PubMed]

18. Edgar, R.C. MUSCLE: A multiple sequence alignment method with reduced time and space complexity. BMC Bioinformat. 2004, 5, 113. [CrossRef] [PubMed]

19. Altschul, S.F.; Gish, W.; Miller, W.; Myers, E.W.; Lipman, D.J. Basic local alignment search tool. J. Mol. Biol. 1990, 215, 403-410. [CrossRef]

20. Letunic, I.; Bork, P. Interactive Tree Of Life v2: Online annotation and display of phylogenetic trees made easy. Nucleic Acids Res. 2011, 39, 475-478. [CrossRef] [PubMed]

21. Kim, D.; Pertea, G.; Trapnell, C.; Pimentel, H.; Kelley, R.; Salzberg, S.L. TopHat2: Accurate alignment of transcriptomes in the presence of insertions, deletions and gene fusions. Genome Biol. 2013, 25, R36. [CrossRef] [PubMed]

22. Anders, S.; Pyl, P.T.; Huber, W. HTSeqa Python framework to work with high-throughput sequencing data. Bioinformatics 2015, 31, 166-169. [CrossRef] [PubMed]

23. Czechowski, T.; Stitt, M.; Altmann, T.; Udvardi, M.K.; Scheible, W.R. Genome-wide identification and testing of superior reference genes for transcript normalization in Arabidopsis. Plant Physiol. 2005, 139, 5-17. [CrossRef] [PubMed]

(C) 2017 by the authors. Licensee MDPI, Basel, Switzerland. This article is an open access article distributed under the terms and conditions of the Creative Commons Attribution (CC BY) license (http://creativecommons.org/licenses/by/4.0/). 